\title{
Optimal Time-varying P-controller for a Class of Uncertain Nonlinear Systems
}

\author{
M. Suruz Miah and Wail Gueaieb
}

\begin{abstract}
In this manuscript, an optimal time-varying P-controller is presented for a class of continuous-time underactuated nonlinear systems in the presence of process noise associated with systems' inputs. This is a state feedback control strategy where the optimization is performed on a time-varying feedback operator (herein called the feedback control gain). The main goal of the current manuscript is to provide a framework for multi-input multi-output nonlinear systems which yields a satisfactory tracking performance based on the optimal time-varying feedback control gain. Unlike other feedback control techniques that perform dynamic linearization of system models, the proposed time-varying P-controller provides the full-state feedback control to the original nonlinear system model. Hence, this P-controller guarantees global asymptotic statetracking. Furthermore, the bounded system's process noise is taken into consideration to measure the controller's robustness. The proposed P-controller is tested for its nonlinear trajectory tracking and fixed-point stabilization capabilities with two nonholonomic systems in the presence of actuators' noise.
\end{abstract}

Keywords: Feedback control gain, Gradient descent, Hamiltonian, Lagrange multipliers, Mobile robots, Optimal control, Robustness.

\section{INTRODUCTION}

Feedback control design for tracking a pre-defined trajectory or stabilizing to a fixed point using a nonlinear underactuated system is a quite challenging task. The tracking problem is usually divided into two sub-problems: state tracking and output tracking, which deal with the stabilization of the system outputs or states to any reference output or desired state (especially at an equilibrium point) [14]. The stabilization problem has been extensively studied for both linear and nonlinear systems due to its relative simplicity. However, the tracking problem of nonlinear systems still stands as a real challenge, especially in the face of uncertainties [5]. As such, practical alternative control solutions that guarantee acceptable tracking performance for systems with input uncertainties are well motivated. This manuscript contributes to the development of an optimal time-varying P-control law for a class of nonlinear systems in presence of state-dependent process noise, which guarantees global asymptotic statetracking.

Several control laws have been proposed in [6-9], which are based on basic optimal control theory. A large body of research work has been conducted on Model Predictive Control (MPC) schemes that rely on the solution of an open-loop optimal control problem to predict the system behavior over a time horizon [10-15]. More specifically,

M. Suruz Miah and Wail Gueaieb are with the School of Electrical Engineering and Computer Science, University of Ottawa, Ottawa, Ontario, K1N 6N5, Canada (e-mail: suruz.miah@uOttawa.ca and wgueaieb@eecs.uOttawa.ca). robust MPC strategies have been proposed to solve the stabilization problem of nonholonomic systems in the presence of model uncertainty [16 17]. The authors in [18] designed and analyzed controllers for Lipschitzian nonlinear systems to eventually derive an adequate observer for such a class of systems. In order to address stabilization performance, robustness, actuator fault tolerance and disturbance attenuation of dynamic systems, LMI-based linear state feedback has been used extensively [19]. Such techniques are based on a nonlinearity assumption, in the form of $f(x)=0$ at $x=0$, for example. Although this makes the problem handling easier, the issue of stabilization of classes of systems not satisfying this assumption remains open [5]. Some non-conventional control laws, such as those using neural network and fuzzy control logic, for instance, have been proposed to resolve this problem. These control strategies, however, suffer from their relatively high computational complexity and the time-consuming manual parameter tuning. In addition, in most cases they were only applicable to single-input-single-output (SISO) nonlinear systems (see [20-22]). The authors in [23, 24] presented output feedback control laws for a class of uncertain linear dynamical systems. These laws either assume a linear system model or the existence of feasible control inputs (reference control) satisfying certain objectieve functions for the system to be controlled. Some geometric control techniques such as differential flatness and backstepping have been applied as well [25, 26]. These techniques have been witnessed to have a satisfactory tracking performance when applied to affine nonlinear systems, but 
they require quite complex feedback laws even for simple systems, such as a unicycle, for example. A recent and interesting global optimization approach coupled with the support vector machine technique has been applied to optimize motion of robotic manipulators [27]. This idea has been extended in the current manuscript through optimizing a time-varying feedback gain, which does not rely on sampling-based methods as in [27].

Despite the aforementioned contributions of the control community, the trajectory tracking problem for uncertain control-affine nonlinear systems still faces significant technical challenges, (such as the non-existence of smooth time-invariant feedback laws, relying on model linearization and initial perturbations, for example). This paper addresses the design of a time-varying P-controller for nonholonomic systems in the presence of bounded state dependent noise associated with the systems' inputs. The proposed controller solves, both, the stabilization and trajectory tracking problems in an unified manner. Instead of optimizing the control inputs directly, the proposed controller optimizes a time-varying feedback operator using a gradient-based update rule. The approach is different from most of the controllers suggested in the literature in that it optimizes a general feedback control gain (feedback operator) which eventually provides the optimal control inputs to the system. Unlike similar counterparts [28-30], it does not require any linearization of the system model.

The rest of the manuscript is outlined as follows. Mathematical preliminaries are presented in section 2. In section 3. the system model and problem statement are defined. The main contribution of this paper, which is the design of the feedback control gain (optimal P-controller), is illustrated in section 4. The robustness of the proposed controller is demonstrated in section 5. A thorough evaluation of the control strategy is carried out through numerical simulations on two nonlinear systems. The results are presented and discussed in section 6. Finally, conclusions are drawn in section 7 .

\section{PRELIMINARIES}

Throughout this paper, scalar quantities will be denoted by lower-case letters, while vectors will be denoted by lower-case bold letters. Upper-case bold letters will denote matrices. For any positive integer $n, \mathbb{R}^{n}$ denotes the Euclidean space. For any vector $\mathbf{x}, \mathbf{y} \in \mathbb{R}^{n}$, the 2-norm and the scalar product are given by

$$
\|\mathbf{x}\| \equiv\left[\sum_{i=1}^{n}\left|x_{i}\right|^{2}\right]^{1 / 2} \text { and }(\mathbf{x} \cdot \mathbf{y}) \equiv \mathbf{x}^{T} \mathbf{y} \equiv \sum_{i=1}^{n} x_{i} y_{i}
$$

respectively. We shall use $\mathbb{R}^{m \times n}$ to denote the space of $m \times n$ matrices with entries from $\mathbb{R}$. Let $\mathbf{X}, \mathbf{Y} \in \mathbb{R}^{m \times n}$, then the 2-norm and their scalar product are also given by

$$
\|\mathbf{X}\| \equiv\left[\sum_{i=1}^{m} \sum_{j=1}^{n}\left|x_{i, j}\right|^{2}\right]^{1 / 2},(\mathbf{X} \cdot \mathbf{Y}) \equiv \operatorname{Tr}\left[\mathbf{X}^{T} \mathbf{Y}\right] \equiv \operatorname{Tr}\left[\mathbf{X} \mathbf{Y}^{T}\right],
$$

respectively, where $\operatorname{Tr}[\cdot]$ denotes the trace of matrix [.]. Clearly, $\operatorname{Tr}\left[\mathbf{X}^{T} \mathbf{X}\right]=\|\mathbf{X}\|^{2}$. If the function $J: \mathbb{R}^{n} \rightarrow \mathbb{R}$ is differentiable at $\mathbf{x} \in \mathbb{R}^{n}$, then for any $v \in \mathbb{R}^{n}, d J(\mathbf{x} ; v)$ denotes the Gateaux (directional) derivative in the direction of $v$, which is given by

$$
d J(\mathbf{x} ; v)=v^{T} \nabla J=\lim _{\varepsilon \rightarrow 0} \frac{J(\mathbf{x}+\varepsilon v)-J(\mathbf{x})}{\varepsilon},
$$

where $\nabla J$ denotes the gradient of $J$. However, if $J: \mathbb{R}^{m \times n} \rightarrow$ $\mathbb{R}$, then for any $\mathbf{X}, \mathbf{V} \in \mathbb{R}^{m \times n}$, the directional derivative in the direction of $\mathbf{V}$ is defined by

$$
d J(\mathbf{X} ; \mathbf{V})=\operatorname{Tr}\left[\mathbf{V}^{T} \nabla J\right]=\lim _{\varepsilon \rightarrow 0} \frac{J(\mathbf{X}+\varepsilon \mathbf{V})-J(\mathbf{X})}{\varepsilon} .
$$

If $t_{0}$ and $t_{f}$ denote the initial and final time, respectively, with $0 \leq t_{0}<t \leq t_{f}<\infty$; and $\mathscr{I} \equiv\left[t_{0}, t_{f}\right]$ denotes the finite time interval, then $C\left(\mathscr{I}, \mathbb{R}^{n}\right)$ denotes the class of all continuous functions on $\mathscr{I}$ taking values in $\mathbb{R}^{n}$. Let $p \in[1, \infty)$ and $\mathscr{I}$ be any finite time interval, we use $\mathscr{L}_{p}\left(\mathscr{I}, \mathbb{R}^{n}\right)$ to denote the set of Lebesgue measurable functions $\{\mathbf{f}\}$ defined on $\mathscr{I}$ and taking values in $\mathbb{R}^{n}$ whose norms are $p$-th power integrable i.e.,

$$
L_{p}(\mathbf{f})=\left(\int_{t_{0}}^{t_{f}}\|\mathbf{f}\|^{p} d t\right)^{1 / p}<\infty
$$

where $L_{p}(\mathbf{f})$ denotes the $p$-th norm of the function $\mathbf{f} 31$. 32]. For $p=\infty, \mathscr{L}_{\infty}\left(\mathscr{I}, R^{n}\right)$ denotes the space of Lebesgue measurable functions $\{\mathbf{f}\}$ defined on $\mathscr{I}$ and taking values in $\mathbb{R}^{n}$ satisfying ess $\sup \{\|\mathbf{f}(t)\|, t \in \mathscr{I}\}<\infty$. The notation $\operatorname{diag}\left(a_{1}, \ldots, a_{n}\right)$ is used to represent a diagonal matrix whosewith diagonal entries $a_{1}, \ldots, a_{n}$ and $\mathscr{N}$ denotes the set of natural numbers.

\section{SYSTEM MODEL AND PROBLEM STATEMENT}

For positive integers, $n, m, s$, with $n>m \geq s$, and the time interval $\mathscr{I} \equiv\left[t_{0}, t_{f}\right], 0 \leq t_{0}<t_{f}$, consider a family of nonlinear systems given by

$$
\dot{\mathbf{x}}(t)=\mathbf{g}_{0}[t, \mathbf{x}(t)]+\sum_{j=1}^{m} \mathbf{g}_{j}[t, \mathbf{x}(t)] u_{j}(t)+\mathbf{G}[t, \mathbf{x}(t)] \xi(t)
$$

where $\mathbf{x}(t) \in \mathbb{R}^{n}$ is the system state, $t \in \mathscr{I}$, the control input $\mathbf{u}(t)=\left[u_{1}(t), u_{2}(t), \ldots, u_{m}(t)\right]^{T} \in \mathbb{R}^{m}, \xi(t) \in \mathbb{R}^{s}$ is the noise vector associated with the control inputs $\mathbf{u}(t)$, $\mathbf{g}_{0}, \mathbf{g}_{j}: \mathbb{R}^{n} \rightarrow \mathbb{R}^{n}, \mathbf{G}: \mathscr{I} \times \mathbb{R}^{n} \rightarrow \mathbb{R}^{n \times s}$, and $\mathbf{x}\left(t_{0}\right)=\mathbf{x}_{0}$ is the system's initial state. 
Let $\mathbf{x}^{d}(t)$ and $\mathbf{u}^{d}(t)$ be the desired (reference) trajectory and the desired input, respectively. The desired reference input can be written as

$$
\dot{\mathbf{x}}^{d}(t)=\mathbf{g}_{0}\left[t, \mathbf{x}^{d}(t)\right]+\sum_{j=1}^{m} \mathbf{g}_{j}\left[t, \mathbf{x}^{d}(t)\right] u_{j}^{d}(t)
$$

Suppose $\mathbf{e}(t)=\mathbf{x}(t)-\mathbf{x}^{d}(t)$ denotes the tracking error, for $t \in \mathscr{I}$. The objective is to find the optimal admissible control input $\mathbf{u}(t) \in \mathscr{U}_{a d} \subset \mathbb{R}^{m}$ that generate the state trajectory $\mathbf{x}(t) \in \mathscr{X} \subset \mathbb{R}^{n}$ while minimizing the average cumulative tracking error, $\mathscr{E}_{\text {avg }}$, given by

$$
\mathscr{E}_{\text {avg }}=\frac{1}{t_{f}-t_{0}} \int_{t_{0}}^{t_{f}} \mathbf{e}(t) d t, \quad t_{0} \neq t_{f}
$$

In the presence of system's input noise and input constraints, the problem can be stated as

$$
\inf _{\{\mathbf{x} \in \mathscr{X}, \mathbf{u} \in \mathscr{U} a d, \forall t \in \mathscr{I}\}}\left[\mathscr{E}_{\text {avg }}\right]
$$

\section{OPTIMAL P-CONTROL LAW}

This section presents the optimal time-varying P-control law, which is the main contribution of this manuscript. Throughout this manuscript, we introduce the following assumptions.

Assumption 1: The vector field $\mathbf{g}_{j}[\mathbf{x}(t)]$, for $j=0, \ldots, m$, is globally Lipschitz and satisfies

$\left\|\mathbf{g}_{j}[\mathbf{x}(t)]-\mathbf{g}_{j}[\mathbf{y}(t)]\right\| \leq L_{c}\|\mathbf{x}(t)-\mathbf{y}(t)\|, \forall \mathbf{x}, \mathbf{y} \in \mathbb{R}^{n}, t \in \mathscr{I}$,

where $L_{c}$ is the Lipschitz constant.

Assumption 2: The noise process $\xi:[0, \infty) \longrightarrow R^{s}$ is any measurable (Lebesgue integrable) stochastic process taking values from the closed ball $\mathscr{B}\left(\bar{\xi}, r_{d}\right)$ defined by

$$
\mathscr{B}\left(\bar{\xi}, r_{d}\right)=\left\{\xi(t) \in \mathbb{R}^{s}:\|\xi(t)-\bar{\xi}\| \leq r_{d}\right\},
$$

where $r_{d}>0$ is the radius of the noise associated with the system's inputs and $\bar{\xi}$ is the mean of $\xi(t)$, for $t \in \mathscr{I}$.

We do not assume any probabilistic structure for the process $\{\xi\}$ except that it is a measurable process and essentially bounded and hence locally square integrable [33]. As such, the total energy in the noise is $\int_{t_{0}}^{t_{f}}\|\xi(t)\|^{2} d t \leq$ $r_{d}^{2}\left(t_{f}-t_{0}\right)$.

Assumption 3: System (1) has constraint on its input as

$$
\left|u_{j}(t)\right| \leq u^{\max }, \text { for } j=1, \ldots, m, t \in \mathscr{I},
$$

where $u^{\max }$ is the upper bound of the systems' inputs. In other words, $\mathbf{u}(t)$ must be chosen from a set of admissible controls, $\mathscr{U}_{a d}$, i.e., $\mathbf{u}(t) \in \mathscr{U}_{a d} \subset \mathbb{R}^{m}$ which are used to generate the set of admissible state trajectories $\mathscr{X}$.
Without loss of generality, let us assume that the control input $\mathbf{u}$ and the noise vector $\xi$ have the same dimensions, i.e., $m=s$. Defining the input error as $\tilde{\mathbf{u}}(t)=\mathbf{u}(t)-$ $\mathbf{u}^{d}(t)$, the error dynamics can easily be derived from models (1) and (2), which is given by

$$
\begin{aligned}
& \dot{\mathbf{e}}=\mathbf{g}_{0}\left[t, \mathbf{e}(t)+\mathbf{x}^{d}(t)\right]-\mathbf{g}_{0}\left[t, \mathbf{x}^{d}(t)\right]+ \\
& \quad\left\{\mathbf{G}\left[t, \mathbf{e}(t)+\mathbf{x}^{d}(t)\right]-\mathbf{G}\left[t, \mathbf{x}^{d}(t)\right]\right\}\left\{\tilde{\mathbf{u}}(t)+\mathbf{u}^{d}(t)\right\} \\
& +\mathbf{G}\left[t, \mathbf{x}^{d}(t)\right] \tilde{\mathbf{u}}(t)+\mathbf{G}\left[t, \mathbf{e}(t)+\mathbf{x}^{d}(t)\right] \xi(t) .
\end{aligned}
$$

The optimal P-control law is defined as

$$
\tilde{\mathbf{u}}(t)=\mathbf{K}_{P}(t) \mathbf{e}(t),
$$

subject to (6), where $\mathbf{K}_{P}(t) \in \mathbb{R}^{m \times n}$ is the feedback control gain for the system (7). $\mathbf{K}_{P}(t)$ must be chosen from a bounded matrix set $\mathscr{K} \subset \mathbb{R}^{m \times n}$. Furthermore, due to the constraint on the system inputs, $\mathbf{K}_{P}(t)$ has to be chosen from the admissible matrix space $\mathscr{K}_{a d} \subset \mathscr{K}$.

Substituting (8) in (7), yields the following full-state feedback system:

$$
\begin{aligned}
\dot{\mathbf{e}}(t) & =\mathbf{f}_{0}[t, \mathbf{e}(t)]+\mathbf{f}_{1}\left[t, \mathbf{e}(t), \mathbf{K}_{P}(t)\right]+\mathbf{f}_{2}[t, \mathbf{e}(t), \xi(t)] \\
& \equiv \mathbf{f}\left[t, \mathbf{e}(t), \mathbf{K}_{P}(t), \xi(t)\right],
\end{aligned}
$$

with $\mathbf{e}\left(t_{0}\right)=\mathbf{e}_{0}$, where

$$
\begin{aligned}
\mathbf{f}_{0}[t, \mathbf{e}(t)] & \equiv \mathbf{g}_{0}\left[t, \mathbf{e}(t)+\mathbf{x}^{d}(t)\right]-\mathbf{g}_{0}\left[t, \mathbf{x}^{d}(t)\right], \\
\mathbf{f}_{1}\left[t, \mathbf{e}(t), \mathbf{K}_{P}(t)\right] & \equiv\left\{\mathbf{G}\left[t, \mathbf{e}(t)+\mathbf{x}^{d}(t)\right]-\mathbf{G}\left[t, \mathbf{x}^{d}(t)\right]\right\} \times \\
& \left\{\mathbf{K}_{P}(t) \mathbf{e}(t)+\mathbf{u}^{d}(t)\right\}+\mathbf{G}\left[t, \mathbf{x}^{d}(t)\right] \mathbf{K}_{P}(t) \mathbf{e}(t), \\
\mathbf{f}_{2}[t, \mathbf{e}(t), \xi(t)] & \equiv \Theta[t, \mathbf{e}(t)] \xi(t) \equiv \mathbf{G}\left[t, \mathbf{e}(t)+\mathbf{x}^{d}(t)\right] \xi(t) .
\end{aligned}
$$

Lemma 1 (Solutions of feedback system): Given assumptions (1)-(3), for every initial condition $\mathbf{e}\left(t_{0}\right) \in \mathbb{R}^{n}$, and the feedback control gain $\mathbf{K}_{P}(t) \in \mathscr{K}_{a d}$, the system (9) has a unique absolutely continuous solution $\mathbf{e}(t) \in C\left(\mathscr{I}, \mathbb{R}^{n}\right)$. Furthermore, the solution set

$\mathscr{E} \equiv\left\{\mathbf{e}(t) \equiv \mathbf{e}\left[t, \mathbf{K}_{P}(t), \xi(t)\right] \in C\left(\mathscr{I}, \mathbb{R}^{n}\right): \mathbf{K}_{P}(t) \in \mathscr{K}_{a d}\right\}$

is a bounded subset of $C\left(\mathscr{I}, \mathbb{R}^{n}\right)$.

The proof of this Lemma is classical and follows from a similar technique in [34, p. 89]. Lemma1 follows that the actual trajectory of the feedback system (9) can be symbolically represented by

$$
\mathbf{e}(t)=\mathbf{e}\left(t_{0}\right)+\int_{t_{0}}^{t} \mathbf{f}\left[\tau, \mathbf{e}(\tau), \mathbf{K}_{P}(\tau), \xi(\tau)\right] d \tau, t \in \mathscr{I} .
$$

In order to solve the problem (4), let us introduce the quadratic cost functional as

$$
\begin{aligned}
J\left(\mathbf{K}_{P}, \xi\right) & =\frac{1}{2}\left\{\mathbf{e}^{T}\left(t_{f}\right) \mathbf{P}\left(t_{f}\right) \mathbf{e}\left(t_{f}\right)+\int_{t_{0}}^{t_{f}} \mathbf{e}^{T}(t) \mathbf{Q}(t) \mathbf{e}(t) d t\right\} \\
& \equiv \phi\left[t_{f}, \mathbf{e}\left(t_{f}\right)\right]+\int_{t_{0}}^{t_{f}} \ell[t, \mathbf{e}(t)] d t,
\end{aligned}
$$


where $\mathbf{P}\left(t_{f}\right) \in \mathbb{R}^{n \times n}$ and $\mathbf{Q}(t) \in \mathbb{R}^{n \times n}$ are symmetric positive definite matrices that indicate the relative importance of the error components along $\mathbb{R}^{n}$. The performance index $J\left(\mathbf{K}_{P}, \xi\right)$ in (11) depends on the feedback control gain matrix $\mathbf{K}_{P}(t)$ and noise vector $\xi(t)$ through the state variable $\mathbf{e}(t)$ as it is clear from the feedback system (9).

The task now becomes solving the following regulator problem:

$$
\inf _{\left\{\mathbf{K}_{P} \in \mathscr{K}_{a d}, \boldsymbol{\xi} \in \mathscr{B}\left(\bar{\xi}, r_{d}\right)\right\}}\left[J\left(\mathbf{K}_{P}, \xi\right)\right],
$$

which yields $\mathbf{e}(t) \rightarrow \mathbf{0}$, for $t \in \mathscr{I}$. Note that solving the problem (12) will eventually solve the problem (4). This point will be clearer later. Hence, the problem (12) can be solved if there exists an optimal time-varying feedback gain $\mathbf{K}_{P}^{*}(t)$, for $t \in \mathscr{I}$.

Theorem 1 (Existence of optimal feedback gain $\mathbf{K}_{P}^{*}(t)$ ): For the feedback system (9), suppose the basic assumptions (17)-(3) hold. For any fixed noise $\xi_{c} \in \mathscr{B}\left(\bar{\xi}, r_{d}\right)$, there exists an optimal time-varying feedback control gain $\mathbf{K}_{P}^{*}(t) \in \mathscr{K}_{a d}$ that solves the regulator problem (12).

Proof: Using the well known Alaoglu's theorem, $\mathscr{K}_{\text {ad }} \subset$ $\mathscr{K} \subset \mathbb{R}^{m \times n}$ is a (weak star) $w^{*}$ compact set and it suffices to prove that $\mathbf{K}_{P} \longmapsto J\left(\mathbf{K}_{P}, \xi_{c}\right)$ is sequentially weak star continuous. Let $\left\{\mathbf{K}_{P}^{i}, i \in \mathscr{N}\right\} \in \mathscr{K}_{\text {ad }}$ be a sequence and suppose $\mathbf{K}_{P}^{i} \stackrel{w^{*}}{\longmapsto} \mathbf{K}_{P}^{*}$. Since $\mathscr{K}_{a d}$ is $w^{*}$ closed, we have $\mathbf{K}_{P}^{*} \in \mathscr{K}_{\text {ad }}$ [33].

For simplicity of the proof, we suppress the variable $t$ for clarity. Let $\left\{\mathbf{e}^{i}, i \in \mathscr{N}\right\}$ and $\mathbf{e}^{*}$ denote the solutions of the system (9) corresponding to $\left\{\mathbf{K}_{P}^{i}, i \in \mathscr{N}\right\}$ and $\mathbf{K}_{P}^{*}$, respectively. Hence, the corresponding state equation becomes

$$
\dot{\mathbf{e}}^{i}=\mathbf{f}\left(\mathbf{e}^{i}, \mathbf{K}_{P}^{i}, \xi_{c}\right) \text { and } \dot{\mathbf{e}}^{*}=\mathbf{f}\left(\mathbf{e}^{*}, \mathbf{K}_{P}^{*}, \xi_{c}\right), \mathbf{e}_{0}^{i}=\mathbf{e}_{0}^{*}=\mathbf{e}_{0} .
$$

Using [10], the solutions of the above two state-space model can be described by

$$
\begin{aligned}
\mathbf{e}^{i}(t) & =\mathbf{e}_{0}+\int_{t_{0}}^{t} \mathbf{f}\left[\mathbf{e}^{i}(\tau), \mathbf{K}_{P}^{i}(\tau), \xi_{c}(\tau)\right] d \tau, \text { and } \\
\mathbf{e}^{*}(t) & =\mathbf{e}_{0}+\int_{t_{0}}^{t} \mathbf{f}\left[\mathbf{e}^{*}(\tau), \mathbf{K}_{P}^{*}(\tau), \xi_{c}(\tau)\right] d \tau .
\end{aligned}
$$

Subtracting one from another, we get

$$
\begin{aligned}
& \mathbf{e}^{i}(t)-\mathbf{e}^{*}(t)= \\
& \int_{0}^{t}\left\{\mathbf{f}\left[\mathbf{e}^{i}(\tau), \mathbf{K}_{P}^{i}(\tau), \xi_{c}(\tau)\right]-\mathbf{f}\left[\mathbf{e}^{*}(\tau), \mathbf{K}_{P}^{*}(\tau), \xi_{c}(\tau)\right]\right\} d \tau .
\end{aligned}
$$

Taking the Euclidean norm in both sides of the above expression and using the triangle inequality yield

$$
\left\|\mathbf{e}^{i}(t)-\mathbf{e}^{*}(t)\right\| \leq \mathbf{v}^{i}(t)+\int_{t_{0}}^{t} L_{\beta}(\tau)\left\|\mathbf{e}^{i}(\tau)-\mathbf{e}^{*}(\tau)\right\| d \tau,
$$

where $\mathbf{v}^{i}(t)$ can be symbolically represented as

$$
\mathbf{v}^{i}(t)=\left\|\int_{\mathscr{I}}\left[\int_{\mathscr{K}} \mathbf{f}\left(\mathbf{e}^{*}(\tau), \Gamma, \xi_{c}\right)\left(\mathbf{K}_{P}^{i}(d \Gamma)-\mathbf{K}_{P}^{*}(d \Gamma)\right)\right] d \tau\right\|,
$$

for $\Gamma \in \mathscr{K}$ and $L_{\beta}(t) \in \mathscr{L}_{1}^{+}(\mathscr{I})$ (see Theorem 8.3.4 of [34 p. 273] for more details). Thus, it follows from Gronwall inequality that

$$
\begin{aligned}
& \left\|\mathbf{e}^{i}(t)-\mathbf{e}^{*}(t)\right\| \leq \mathbf{v}^{i}(t)+ \\
& \quad \int_{t_{0}}^{t} \exp \left\{\int_{\tau}^{t} L_{\beta}\left(\tau_{1}\right) d \tau_{1}\right\} L_{\beta}(\tau) \mathbf{v}^{i}(\tau) d \tau .
\end{aligned}
$$

Clearly, $v^{i}(t) \rightarrow 0$, for $t \in \mathscr{I}, i \in \mathscr{N}$, as $\mathbf{K}_{P}^{i} \stackrel{w^{*}}{\longmapsto} \mathbf{K}_{P}^{*}$. Thus, it follows from inequality (13) that $\mathbf{e}^{i} \stackrel{\mathbf{K}_{P}}{\longmapsto} \mathbf{e}^{*}$.

Since both $\ell(t, \cdot)$ and $\phi(t, \cdot)$ are continuous on $\mathbb{R}^{n}$, we have $\ell\left[t, \mathbf{e}_{i}(t)\right] \longrightarrow \ell\left[t, \mathbf{e}^{*}(t)\right]$ for almost all $t \in \mathscr{I}$ and $\phi\left[t, \mathbf{e}^{i}\left(t_{f}\right)\right]$ $\longrightarrow \phi\left[t, \mathbf{e}^{*}\left(t_{f}\right)\right]$ as $i \rightarrow \infty$. Thus, it follows from the expression (11) that $\lim _{i \rightarrow \infty} J\left(\mathbf{K}_{P}^{i}, \xi_{c}\right)=J\left(\mathbf{K}_{P}^{*}, \xi_{c}\right)$ proving weak star continuity of $J$ on $\mathscr{K}_{a d}$. Since $\mathscr{K}_{a d}$ weak star compact, $J$ attains its minimum on $\mathscr{K}_{a d}$.

Theorem 1 guarantees that there exists an optimal feedback gain $\mathbf{K}_{P}^{*}$ for the system (9). In the following, we drop the argument $(t)$ when no ambiguity arises. To solve for the optimal trajectory that minimizes the objective functional (11), we need to derive the necessary conditions of optimality. These necessary conditions are most readily found if the integrand of the cost functional 111 is recast in terms of Hamiltonian $\mathscr{H}: \mathscr{I} \times \mathbb{R}^{n} \times \mathbb{R}^{n} \times \mathbb{R}^{m \times n} \longrightarrow \mathbb{R}$, which is expressed by

$$
\begin{aligned}
& \mathscr{H}\left[t, \mathbf{e}, \phi, \mathbf{K}_{P}\right]=\phi^{T} \mathbf{f}_{0}(t, \mathbf{e})+ \\
& \phi^{T}\left\{\mathbf{f}_{1}\left(t, \mathbf{e}, \mathbf{K}_{P}\right)+\mathbf{f}_{2}(t, \mathbf{e}, \xi)\right\}+\ell(t, \mathbf{e}),
\end{aligned}
$$

where $\phi \in \mathbb{R}^{n}, t \in \mathscr{I}$, is a vector of Lagrange multipliers whose elements are the co-states of the system.We now derive the necessary conditions of optimality for the error feedback model (9).

Theorem 2 (Necessary Conditions of Optimality): Based on Theorem 11 it follows that $\mathbf{e}(t) \stackrel{\mathbf{K}_{P}^{*}(t)}{\longrightarrow} \mathbf{e}^{*}(t)$ and the optimal error trajectory $\mathbf{e}^{*}(t), t \in \mathscr{I}$ for the feedback model 9 can be obtained if there exists an optimal multiplier $\phi^{*}(t) \in$ $C\left(\mathscr{I}, \mathbb{R}^{n}\right)$ such that the triple $\left\{\mathbf{e}^{*}, \phi^{*}, \mathbf{K}_{P}^{*}\right\}$ satisfies the following necessary conditions:

$$
\begin{gathered}
\left.\mathscr{H} t, \mathbf{e}^{*}, \phi^{*}, \mathbf{K}_{P}\right) \geq \mathscr{H}\left(t, \mathbf{e}^{*}, \phi^{*}, \mathbf{K}_{P}^{*}\right), \mathbf{K}_{P} \in \mathscr{K}, \\
\dot{\mathbf{e}}^{*}=\frac{\partial \mathscr{H}}{\partial \phi}\left(t, \mathbf{e}^{*}, \phi^{*}, \mathbf{K}_{P}^{*}\right), \mathbf{e}^{*}\left(t_{0}\right)=\mathbf{e}_{0}, \\
\dot{\phi}^{*}=-\frac{\partial \mathscr{H}}{\partial \mathbf{e}}\left(t, \mathbf{e}^{*}, \phi^{*}, \mathbf{K}_{P}^{*}, \phi^{*}\left(t_{f}\right)=\frac{\partial \phi}{\partial \mathbf{e}}\left[t_{f}, \mathbf{e}\left(t_{f}\right)\right] .\right.
\end{gathered}
$$

Proof: Let $\mathbf{e}(t) \equiv \mathbf{e}\left[t, \mathbf{K}_{P}(t), \xi(t)\right]$ be the solution of the feedback system (9), with the cost functional (11) for any choice of $\mathbf{K}_{P}(t) \in \mathscr{K}_{a d}$. For simplicity of the proof, and without loss of generality, assume that the noise vector $\xi(t) \equiv \xi_{c}$ is fixed. Since $\mathbf{K}_{P}^{*}(t)$ is optimal with the associated trajectory $\mathbf{e}^{*}(t)$, it is clear that

$$
J\left(\mathbf{K}_{P}^{*}, \xi_{c}\right) \leq J\left(\mathbf{K}_{P}, \xi_{c}\right), \forall \mathbf{K}_{P}(t) \in \mathscr{K}_{a d}, t \in \mathscr{I} .
$$


Suppressing the variable $(t)$ for clarity and for any $\varepsilon \in$ $[0,1]$, we define $\mathbf{K}_{P}^{\varepsilon}=\mathbf{K}_{P}^{*}+\varepsilon\left(\mathbf{K}_{P}-\mathbf{K}_{P}^{*}\right)$. Since $\mathscr{K}_{P}$ is a closed convex set, $\mathscr{K}_{a d}$ is also a closed convex subset of $\mathscr{L}_{\infty}\left(I, \mathbb{R}^{m \times n}\right)$ and therefore $\mathbf{K}_{P}^{\varepsilon} \in \mathscr{K}_{a d}$. Thus $J\left(\mathbf{K}^{*}, \boldsymbol{\xi}_{c}\right) \leq$ $J\left(\mathbf{K}_{P}^{\varepsilon}, \xi_{c}\right)$, which follows that

$d J\left(\mathbf{K}_{P}, \xi_{c} ; \mathbf{K}_{P}-\mathbf{K}_{P}^{*}\right)=\operatorname{Tr}\left[\left(\mathbf{K}_{P}-\mathbf{K}_{P}^{*}\right)^{T} \nabla J\left(\mathbf{K}_{P}^{*}, \xi_{c}\right)\right] \geq 0$,

where $d J$ denotes the Gateaux (directional) derivative of $J$ in the direction of $\left(\mathbf{K}_{P}-\mathbf{K}_{P}^{*}\right)$.

Let $\mathbf{e}^{\varepsilon}$ be the solution of the feedback system (9) corresponding to the gain $\mathbf{K}_{P}^{\varepsilon}$ with the same initial state $\mathbf{e}^{\varepsilon}\left(t_{0}\right)=$ $\mathbf{e}_{0}$. It is easy to verify that

$$
\lim _{\varepsilon \rightarrow 0} \mathbf{K}_{P}^{\varepsilon}(t)=\mathbf{K}_{P}^{*}(t), \text { and } \quad \lim _{\varepsilon \rightarrow 0} \mathbf{e}^{\varepsilon}(t)=\mathbf{e}^{*}(t) .
$$

Hence, the state trajectories $\mathbf{e}^{\varepsilon}(t)$ and $\mathbf{e}^{*}(t)$ can be defined as

$$
\begin{aligned}
\dot{\mathbf{e}}^{\varepsilon} & =\mathbf{f}_{0}\left(t, \mathbf{e}^{\varepsilon}\right)+\mathbf{f}_{1}\left(t, \mathbf{e}^{\varepsilon}, \mathbf{K}_{P}^{\varepsilon}\right)+\mathbf{f}_{2}\left(t, \mathbf{e}^{\varepsilon}, \xi_{c}\right) \text { and } \\
\dot{\mathbf{e}}^{*} & =\mathbf{f}_{0}\left(t, \mathbf{e}^{*}\right)+\mathbf{f}_{1}\left(t, \mathbf{e}^{*}, \mathbf{K}_{P}^{*}\right)+\mathbf{f}_{2}\left(t, \mathbf{e}^{*}, \xi_{c}\right), \text { with }
\end{aligned}
$$

$\mathbf{e}^{\varepsilon}\left(t_{0}\right)=\mathbf{e}^{*}\left(t_{0}\right)=\mathbf{e}_{0}$ and $t \in \mathscr{I}$. Subtracting one from the other and by straight forward algebra, one can derive the following equation

$$
\begin{aligned}
\dot{\mathbf{e}}^{\varepsilon}-\dot{\mathbf{e}}^{*}= & \mathbf{f}_{0}\left(t, \mathbf{e}^{\varepsilon}\right)-\mathbf{f}_{0}\left(t, \mathbf{e}^{*}\right)+\mathbf{f}_{1}\left(t, \mathbf{e}^{\varepsilon}, \mathbf{K}_{P}^{*}\right)-\mathbf{f}_{1}\left(t, \mathbf{e}^{*}, \mathbf{K}_{P}^{*}\right)+ \\
& \mathbf{f}_{2}\left(t, \mathbf{e}^{\varepsilon}, \xi_{c}\right)-\mathbf{f}_{2}\left(t, \mathbf{e}^{*}, \xi_{c}\right)+\varepsilon \hat{\mathbf{f}}\left(t, \mathbf{e}^{\varepsilon}, \mathbf{K}_{P}-\mathbf{K}_{P}^{*}\right),
\end{aligned}
$$

where $\hat{\mathbf{f}}\left(t, \mathbf{e}^{\varepsilon}, \mathbf{K}_{P}-\mathbf{K}_{P}^{*}\right)=\mathbf{G}\left(t, \mathbf{e}^{\varepsilon}+\mathbf{x}^{d}\right)\left(\mathbf{K}_{P}-\mathbf{K}_{P}^{*}\right) \mathbf{e}^{\varepsilon}$. Dividing by $\varepsilon$ and denoting

$$
\eta(t) \equiv \lim _{\varepsilon \rightarrow 0}\left(\frac{\mathbf{e}^{\varepsilon}(t)-\mathbf{e}^{*}(t)}{\varepsilon}\right),
$$

it follows from the expression (19) that $\eta(t)$ must satisfy the following initial value problem

$$
\dot{\eta}=\Delta_{\mathbf{e}}\left(t, \mathbf{e}^{*}, \mathbf{K}_{P}^{*}, \xi_{c}\right) \eta(t)+\hat{\mathbf{f}}\left(t, \mathbf{e}^{*}, \mathbf{K}_{P}-\mathbf{K}_{P}^{*}\right),
$$

with $\eta\left(t_{0}\right)=0$, where

$$
\begin{aligned}
& \Delta_{\mathbf{e}}\left(t, \mathbf{e}^{*}, \mathbf{K}_{P}^{*}, \xi_{c}\right)= \\
& \quad \frac{\partial \mathbf{f}_{0}}{\partial \mathbf{e}}\left(t, \mathbf{e}^{*}\right)+\frac{\partial \mathbf{f}_{1}}{\partial \mathbf{e}}\left(t, \mathbf{e}^{*}, \mathbf{K}_{P}^{*}\right)+\frac{\partial \mathbf{f}_{2}}{\partial \mathbf{e}}\left(t, \mathbf{e}^{*}, \xi_{c}\right) .
\end{aligned}
$$

Equation (20) is a linear non-homogeneous equation with $\hat{\mathbf{f}}\left(t, \mathbf{e}^{*}, \mathbf{K}_{P}-\mathbf{K}_{P}^{*}\right)$ being the driving force. As a result, it has a continuous solution $\eta(t) \in C\left(\mathscr{I}, \mathbb{R}^{n}\right)$, which is continuously dependent on $\hat{\mathbf{f}}\left(t, \mathbf{e}^{*}, \mathbf{K}_{P}-\mathbf{K}_{P}^{*}\right)$.

By definition of Gateaux (directional) derivative, we can derive the following expression

$$
\begin{aligned}
& \operatorname{Tr}\left[\left(\mathbf{K}_{P}-\mathbf{K}_{P}^{*}\right)^{T} \nabla J\left(\mathbf{K}_{P}^{*}, \xi_{c}\right)\right]= \\
& \eta^{T}(t) \frac{\partial \phi}{\partial \mathbf{e}}\left[t_{f}, \mathbf{e}\left(t_{f}\right)\right]+\int_{t_{0}}^{t_{f}} \eta^{T}(t) \frac{\partial \ell}{\partial \mathbf{e}}[t, \mathbf{e}(t)] d t .
\end{aligned}
$$

Hence, inequality (18) yields

$$
\eta^{T}(t) \frac{\partial \phi}{\partial \mathbf{e}}\left[t_{f}, \mathbf{e}\left(t_{f}\right)\right]+\int_{t_{0}}^{t_{f}} \eta^{T}(t) \frac{\partial \ell}{\partial \mathbf{e}}[t, \mathbf{e}(t)] d t \geq 0 .
$$

Since $\eta(t)$ of the variational equation 20 is continuously dependent on $\hat{\mathbf{f}}\left(t, \mathbf{e}^{*}, \mathbf{K}_{P}-\mathbf{K}_{P}^{*}\right)$, the map $\hat{\mathbf{f}}\left(t, \mathbf{e}^{\varepsilon}, \mathbf{K}_{P}-\right.$ $\left.\mathbf{K}_{P}^{*}\right) \longrightarrow \eta(t)$ is continuous from $\mathscr{L}_{1}\left(\mathscr{I}, \mathbb{R}^{n}\right)$ to $C\left(\mathscr{I}, \mathbb{R}^{n}\right)$ p. 260]. Hence, the map

$$
\eta(t) \longrightarrow \eta^{T}(t) \frac{\partial \phi}{\partial \mathbf{e}}\left[t_{f}, \mathbf{e}\left(t_{f}\right)\right]+\int_{t_{0}}^{t_{f}} \eta^{T}(t) \frac{\partial \ell}{\partial \mathbf{e}}[t, \mathbf{e}(t)] d t
$$

is a continuous linear functional on $C\left(\mathscr{I}, \mathbb{R}^{n}\right)$. Thus, the composition map

$$
\begin{array}{r}
\hat{\mathbf{f}}\left(t, \mathbf{e}^{*}, \mathbf{K}_{P}-\mathbf{K}_{P}^{*}\right) \longrightarrow \eta^{T}(t) \frac{\partial \phi}{\partial \mathbf{e}}\left[t_{f}, \mathbf{e}\left(t_{f}\right)\right]+ \\
\int_{t_{0}}^{t_{f}} \eta^{T}(t) \frac{\partial \ell}{\partial \mathbf{e}}[t, \mathbf{e}(t)] d t
\end{array}
$$

is a continuous linear functional on $\mathscr{L}_{1}\left(\mathscr{I}, \mathbb{R}^{n}\right)$, where $\hat{\mathbf{f}}\left(t, \mathbf{e}^{\varepsilon}, \mathbf{K}_{P}-\mathbf{K}_{P}^{*}\right) \in \mathscr{L}_{1}\left(\mathscr{I}, \mathbb{R}^{n}\right)$. Therefore, by the Riesz representation theorem or by the duality between $\mathscr{L}_{1}\left(\mathscr{I}, \mathbb{R}^{n}\right)$ and $\mathscr{L}_{\infty}\left(\mathscr{I}, \mathbb{R}^{n}\right)$, we may conclude that there exists an element $\phi^{*} \in \mathscr{L}_{\infty}\left(\mathscr{I}, \mathbb{R}^{n}\right)$ such that

$$
\begin{aligned}
& \operatorname{Tr}\left[\left(\mathbf{K}_{P}-\mathbf{K}_{P}^{*}\right)^{T} \nabla J\left(\mathbf{K}_{P}^{*}, \xi_{c}\right)\right]= \\
& \eta^{T}(t) \frac{\partial \phi}{\partial \mathbf{e}}\left[t_{f}, \mathbf{e}\left(t_{f}\right)\right]+\int_{t_{0}}^{t_{f}} \eta^{T}(t) \frac{\partial \ell}{\partial \mathbf{e}}[t, \mathbf{e}(t)] d t \\
& =\int_{t_{0}}^{t_{f}}\left(\phi^{*}(t)\right)^{T} \hat{\mathbf{f}}\left(t, \mathbf{e}^{*}, \mathbf{K}_{P}-\mathbf{K}_{P}^{*}\right) d t .
\end{aligned}
$$

It follows from the inequality (21) that

$$
\int_{t_{0}}^{t_{f}}\left(\phi^{*}\right)^{T} \hat{\mathbf{f}}\left(t, \mathbf{e}^{*}, \mathbf{K}_{P}-\mathbf{K}_{P}^{*}\right) d t \geq 0, \forall \mathbf{K}_{P} \in \mathscr{K}_{a d} .
$$

Using the variational equation (20), it follows from the second identity of 22) that

$$
\begin{gathered}
\eta^{T}(t) \frac{\partial \phi}{\partial \mathbf{e}}\left[t_{f}, \mathbf{e}\left(t_{f}\right)\right]+\int_{t_{0}}^{t_{f}} \eta^{T}(t) \frac{\partial \ell}{\partial \mathbf{e}}[t, \mathbf{e}(t)] d t= \\
\int_{t_{0}}^{t_{f}}\left\{\left(\phi^{*}\right)^{T}\left[\dot{\eta}-\Delta_{\mathbf{e}}\left(t, \mathbf{e}^{*}, \mathbf{K}_{P}^{*}, \xi_{c}\right) \eta(t)\right]\right\} d t .
\end{gathered}
$$

Integrating by parts and since $\eta\left(t_{0}\right)=0$,

$$
\begin{gathered}
\int_{t_{0}}^{t_{f}}\left(\phi^{*}\right)^{T}\left[\dot{\eta}(t)-\Delta_{\mathbf{e}}\left(t, \mathbf{e}^{*}, \mathbf{K}_{P}^{*}, \xi_{c}\right) \eta(t)\right] d t=\eta^{T}\left(t_{f}\right) \phi^{*}\left(t_{f}\right) \\
+\int_{t_{0}}^{t_{f}} \eta^{T}(t)\left\{-\dot{\phi}^{*}-\Delta_{\mathbf{e}}^{T}\left(t, \mathbf{e}^{*}, \mathbf{K}_{P}^{*}, \xi_{c}\right) \phi^{*}\right\} d t .
\end{gathered}
$$

Expression (24) can now be written as

$$
\begin{aligned}
& \eta^{T}(t) \frac{\partial \phi}{\partial \mathbf{e}}\left[t_{f}, \mathbf{e}\left(t_{f}\right)\right]+\int_{t_{0}}^{t_{f}} \eta^{T}(t) \frac{\partial \ell}{\partial \mathbf{e}}[t, \mathbf{e}(t)] d t= \\
& \eta^{T}\left(t_{f}\right) \phi^{*}\left(t_{f}\right)+\int_{t_{0}}^{t_{f}} \eta^{T}(t)\left\{-\dot{\phi}-\Delta_{\mathbf{e}}^{T}\left(t, \mathbf{e}^{*}, \mathbf{K}_{P}^{*}, \xi_{c}\right) \phi^{*}\right\} d t
\end{aligned}
$$


It is clear from 25 that

$$
\dot{\phi}^{*}=-\Delta_{\mathbf{e}}^{T}\left(t, \mathbf{e}^{*}, \mathbf{K}_{P}^{*}, \xi_{c}\right) \phi^{*}-\frac{\partial \ell}{\partial \mathbf{e}}\left[t, \mathbf{e}^{*}(t)\right],
$$

with $\phi^{*}\left(t_{f}\right)=\frac{\partial \phi}{\partial \mathbf{e}}\left[t_{f}, \mathbf{e}\left(t_{f}\right)\right]$. The co-state dynamics 26 is linear along the optimal error trajectories. Thus, the necessary conditions of optimality is given by the integral inequality (23), the co-state dynamics $(26)$, and the state equation (9). In other words, the choice of $\mathbf{K}_{P} \in \mathscr{K}_{a d}$ determines the optimality conditions $(23),(26)$, and (9).

Consider the optimality condition 23 and rewriting it as follows

$$
\int_{t_{0}}^{t_{f}}\left(\phi^{*}\right)^{T} \hat{\mathbf{f}}\left(t, \mathbf{e}^{*}, \mathbf{K}_{P}\right) d t \geq \int_{t_{0}}^{t_{f}}\left(\phi^{*}\right)^{T} \hat{\mathbf{f}}\left(t, \mathbf{e}^{*}, \mathbf{K}_{P}^{*}\right) d t,
$$

$\forall \mathbf{K}_{P} \in \mathscr{K}_{a d}$. From integral inequality (27), it is easy to derive the point-wise inequality [34]

$$
\left(\phi^{*}\right)^{T} \hat{\mathbf{f}}\left(t, \mathbf{e}^{*}, \mathbf{K}_{P}\right) d t \geq\left(\phi^{*}\right)^{T} \hat{\mathbf{f}}\left(t, \mathbf{e}^{*}, \mathbf{K}_{P}^{*}\right),
$$

$\forall \mathbf{K}_{P} \in \mathscr{K}_{a d}$. Now adding the terms $\left(\phi^{*}\right)^{T} \mathbf{f}_{0}\left(t, \mathbf{e}^{*}\right)$,

$$
\left(\phi^{*}\right)^{T}\left[\mathbf{G}\left(t, \mathbf{e}^{*}+\mathbf{x}^{d}\right)-\mathbf{G}\left(t, \mathbf{x}^{d}\right)\right] \mathbf{u}^{d},\left(\phi^{*}\right)^{T} \mathbf{f}_{2}\left(t, \mathbf{e}^{*}, \xi_{c}\right),
$$

and $\ell\left[t, \mathbf{e}^{*}(t)\right]$ in both sides of (28) yields the Hamiltonian inequality

$$
\mathscr{H}\left[t, \mathbf{e}^{*}(t), \phi^{*}(t), \mathbf{K}_{P}(t)\right] \geq \mathscr{H}\left[t, \mathbf{e}^{*}(t), \phi^{*}(t), \mathbf{K}_{P}^{*}(t)\right] .
$$

This is the same as inequality (15) stated in Theorem 2 Differentiating $\mathscr{H}$ with respect to the co-state variable $\phi$, we get

$$
\frac{\partial \mathscr{H}}{\partial \phi}\left[t, \mathbf{e}^{*}(t), \phi^{*}(t), \mathbf{K}_{P}^{*}(t)\right]=\mathbf{f}\left[t, \mathbf{e}^{*}(t), \mathbf{K}_{P}^{*}(t), \xi_{c}\right],
$$

which leads to the state equation

$$
\dot{\mathbf{e}}^{*}=\frac{\partial \mathscr{H}}{\partial \phi}\left[t, \mathbf{e}^{*}(t), \phi^{*}(t), \mathbf{K}_{P}^{*}(t)\right], \mathbf{e}^{*}\left(t_{0}\right)=\mathbf{e}_{0},
$$

as defined in 16 .

Differentiating $\mathscr{H}$ with respect to the error state variable e yields

$$
\begin{aligned}
\frac{\partial \mathscr{H}}{\partial \mathbf{e}}\left[t, \mathbf{e}^{*}(t), \phi^{*}(t), \mathbf{K}_{P}^{*}(t)\right]= \\
\Delta_{\mathbf{e}}^{T}\left(t, \mathbf{e}^{*}, \mathbf{K}_{P}^{*}, \xi_{c}\right) \phi^{*}+\frac{\partial \ell}{\partial \mathbf{e}}\left[t, \mathbf{e}^{*}(t)\right] .
\end{aligned}
$$

Hence, the co-state dynamics 26 can be expressed in terms of Hamiltonian as

$$
\dot{\phi}^{*}=-\frac{\partial \mathscr{H}}{\partial \mathbf{e}}\left[\mathbf{e}^{*}(t), \phi^{*}(t), \mathbf{K}_{P}^{*}(t)\right], \phi^{*}\left(t_{f}\right)=\frac{\partial \phi}{\partial \mathbf{e}}\left[t_{f}, \mathbf{e}\left(t_{f}\right)\right],
$$

which is condition (17).
Theorem 2 states that the feedback control gain $\mathbf{K}_{P}^{*} \in$ $\mathscr{K}_{a d}$ satisfies the necessary conditions for optimality. In order to solve for $\mathbf{K}_{P}^{*}$, we express the gradient of the Hamiltonian defined in (14) and set it to zero, i.e.,

$$
\mathscr{H}_{\mathbf{K}_{P}} \equiv \frac{\partial \mathscr{H}}{\partial \mathbf{K}_{P}}=\mathbf{0} .
$$

Hence, the problem boils down to finding $\mathbf{K}_{P}(t), t \in \mathscr{I}$, such that the actual error trajectory from $(10)$ and the costate trajectory from (17) satisfy 29]. The optimal feedback control gain $\mathbf{K}_{P}^{*}$ can be determined by satisfying the Hamiltonian inequality (15). In other words, the choice of $\mathbf{K}_{P}$ is to be adaptively tuned to minimize the system's tracking error.

Corollary $\mathbf{1}$ (Adapting the gain $\mathbf{K}_{P}$ ): Consider the feedback system (9) defined over the time horizon $\mathscr{I}$. Adapting the gain $\mathbf{K}_{P}$ according to the following offline update rule

$$
\mathbf{K}_{P}^{\text {new }}=\mathbf{K}_{P}^{\text {old }}-\alpha \mathscr{H}_{\mathbf{K}_{P}}, \text { for } 0<\alpha<1
$$

satisfies the Hamiltonian inequality 15 and, hence, guarantees the convergence of the system's error trajectory to solve the problem 12 .

Proof: Let

$$
\mathbf{K}_{P}^{*}=\mathbf{K}_{P}-\alpha \mathscr{H}_{\mathbf{K}_{P}},
$$

for some $\mathbf{K}_{P} \in \mathbb{R}^{m \times n}$ and $0<\alpha<1$. The corresponding Hamiltonian is $\mathscr{H}\left(t, \mathbf{e}^{*}, \phi^{*}, \mathbf{K}_{P}^{*}\right)=\mathscr{H}\left(t, \mathbf{e}^{*}, \phi^{*}, \mathbf{K}_{P}-\right.$ $\left.\alpha \mathscr{H}_{\mathbf{K}_{P}}\right)$, where $\mathbf{e}^{*}$ and $\phi^{*}$ are the system's error state and the co-state variable corresponding to $\mathbf{K}_{P}^{*}$. Taking the Taylor series expansion of the right hand side about $\mathbf{K}_{P}$, we get

$$
\begin{aligned}
\mathscr{H}\left(t, \mathbf{e}^{*}, \phi^{*}, \mathbf{K}_{P}^{*}\right) & =\mathscr{H}\left(t, \mathbf{e}^{*}, \phi^{*}, \mathbf{K}_{P}\right) \\
& +\operatorname{Tr}\left[\mathscr{H}_{\mathbf{K}_{P}}^{T}\left(\mathbf{K}_{\mathbf{P}}^{*}-\mathbf{K}_{\mathbf{P}}\right)\right]+\mathscr{O}(\alpha) .
\end{aligned}
$$

Neglecting the higher order terms of the above expression yields $\mathscr{H}\left(t, \mathbf{e}^{*}, \phi^{*}, \mathbf{K}_{P}^{*}\right)<\mathscr{H}\left(t, \mathbf{e}^{*}, \phi^{*}, \mathbf{K}_{P}\right)$.

Hence, update rule (31) guarantees that the Hamiltonian $\mathscr{H}$ is monotonically decreasing, which proves that the adaptation law (30) eventually leads to the optimal control operator $\mathbf{K}_{P}^{*}$ yielding the solution of $(12)$. Note that the feedback gain $\mathbf{K}_{P}^{*}(t), t \in \mathscr{I}$, is now used to find the optimal input error signal $\tilde{\mathbf{u}}(t)$ using (8). The actual optimal control input can then be found simply by $\mathbf{u}^{*}(t)=$ $\mathbf{u}^{d}(t)+\tilde{\mathbf{u}}(t)$. This $\mathbf{u}^{*}(t)$ is the solution of (4) which minimizes the average cumulative tracking error defined in (3).

\section{ROBUSTNESS}

This section illustrates the robustness of the feedback control law (8). The robustness result for model (1) presented in this section is an immediate consequence of controlling semi-linear dynamic systems with limited uncertainty illustrated in [33]. We show that the feedback gain 
$\mathbf{K}_{P}^{*}(t)$ is robust with respect to the noise process $\{\xi(t)\}$, $t \in \mathscr{I}$ as long as $\xi(t) \in \mathscr{B}\left(\bar{\xi}, r_{d}\right)$, for $r_{d}>0$. In other words, if we can show that the feedback law (8) is robust for the worst case scenario of the system's process noise $\xi$, then it is robust for all $\xi(t) \in \mathscr{B}\left(\bar{\xi}, r_{d}\right)$.

It is interesting to notice that extreme scenarios correspond to the worst-case situations of the Hamiltonian defined in (14). This happens when the noise vector $\xi$ is co-linear with the vector $\left(\Theta^{T}[t, \mathbf{e}(t)] \phi(t)\right)$ and lies on the boundary of the ball $\mathscr{B}\left(\bar{\xi}, r_{d}\right)$. This is given by the vector $\xi=r_{d} \Upsilon\left(\Theta^{T}[t, \mathbf{e}(t)] \phi(t)\right)$ where the function $\Upsilon: \mathbb{R}^{m} \longrightarrow$ $\mathbb{R}^{m}$ is defined as

$$
\Upsilon(\mathbf{z})=\left\{\begin{array}{ll}
\frac{\mathbf{z}}{\|\mathbf{z}\|}, & \text { if }\|\mathbf{z}\| \neq 0 \\
0, & \text { if }\|\mathbf{z}\|=0 .
\end{array} \quad\right. \text { and }
$$

Considering this worst-case scenario and noting that

$$
\left|\xi^{T} \Theta^{T}[t, \mathbf{e}(t)] \phi(t)\right|_{\mathbb{R}^{m}} \leq r_{d}\left\|\Theta^{T}[t, \mathbf{e}(t)] \phi(t)\right\|_{\mathbb{R}^{m}},
$$

the Hamiltonian in (14) takes the following form

$$
\begin{gathered}
\mathscr{H}\left[t, \mathbf{e}(t), \phi(t), \mathbf{K}_{P}(t)\right]=\phi^{T}(t)\left\{\mathbf{f}_{0}[t, \mathbf{e}(t)]+\mathbf{f}_{1}\left[t, \mathbf{e}(t), \mathbf{K}_{P}(t)\right]\right\} \\
+r_{d}\left\|\Theta^{T}[t, \mathbf{e}(t)] \phi(t)\right\|+\ell[t, \mathbf{e}(t)] .
\end{gathered}
$$

In this case, the state feedback system in (9) becomes

$$
\begin{aligned}
& \dot{\mathbf{e}}(t)=\mathbf{f}_{0}[t, \mathbf{e}(t)]+\mathbf{f}_{1}\left[t, \mathbf{e}(t), \mathbf{K}_{P}(t)\right]+ \\
& \quad r_{d} \Theta[t, \mathbf{e}(t)] \Upsilon\left(\Theta^{T}[t, \mathbf{e}(t)] \phi(t)\right), \mathbf{e}\left(t_{0}\right)=\mathbf{e}_{0} .
\end{aligned}
$$

Hence, the least tracking error in the case of a potentially worst situation can be achieved if the triple $\left\{\mathbf{e}^{*}, \phi^{*}, \mathbf{K}_{P}^{*}\right\}$ satisfies the state feedback model (33), co-state dynamics 26, and the optimality condition (23) simultaneously. One can easily show that the necessary conditions 15 (17) of Theorem 2 can be obtained using the state equation (33) and its corresponding worst case Hamiltonan (32). Moreover, it is important to articulate that instead of considering the random process $\{\zeta(t), t \in \mathscr{I}\}$, in the feedback model (9), one can choose the maximum noise radius $r_{d}$ in the model (33), for which the feedback law (8), with $\mathbf{K}_{P}(t)=\mathbf{K}_{P}^{*}(t)$, guarantees minimizing the average cumulative tracking error defined in (3). In other words, the optimal feedback law (8), with $\mathbf{K}_{P}(t)=\mathbf{K}_{P}^{*}(t)$, minimizes the state tracking errors in the presence of maximum process noise defined by the radius $r_{d}$. This concludes the fact that feedback law $(8)$ is robust in the face of any $\xi(t) \in \mathscr{B}\left(\bar{\xi}, r_{d}\right)$.

In the following, we provide the key steps taken by the controller to numerically solve for the feedback control gain $\mathbf{K}_{P}$, aggregating the components described earlier. $\mathbf{K}_{P}^{i}(t), t \in \mathscr{I}$, denotes the gain at the $i$-th iteration of the optimization procedure.

Step 0 (initialization): Subdivide the time interval $\mathscr{I} \equiv$ $\left[t_{0}, t_{f}\right]$ into $N$ subintervals. Assume a piecewise-contant $\mathbf{K}_{P}^{i}(t)=\mathbf{K}_{P}^{i}\left(t_{k}\right), t \in\left[t_{k}, t_{k+1}\right]$, for $k=0, \ldots, N-1$.
Step 1: Integrate the feedback system (33) with $\mathbf{K}_{P} \equiv$ $\mathbf{K}^{i}(t), t \in \mathscr{I}$.

Step 2: Solve co-state equation (17) backward for $\phi_{i}$.

Step 3: Define the Hamiltonian $\mathscr{H}\left(t, \mathbf{e}_{i}, \phi_{i}, \mathbf{K}_{P}^{i}\right)$ as in (32).

Step 4: Compute the cost function $J\left(\mathbf{K}_{P}^{i}, \xi\right)$ using (11) and $\mathscr{H}_{\mathbf{K}_{P}}$ using 29 .

Step 5: If $J\left(\mathbf{K}_{P}^{i}, \xi\right) \leq \delta$, for a pre-defined small positive tolerance constant $\delta$, then $\mathbf{K}_{P}^{i}$ is regarded close enough to its optimal value, and so the algorithm is halted. Otherwise, use the update rules: $\mathbf{K}_{P}^{i+1}\left(t_{k}\right)=\mathbf{K}_{P}^{i}\left(t_{k}\right)-\alpha \mathscr{H}_{\mathbf{K}_{P}\left(t_{k}\right)}+$ $\lambda \Delta \mathbf{K}_{P}^{i}\left(t_{k}\right)$ and $\Delta \mathbf{K}_{P}^{i}\left(t_{k}\right)=\mathbf{K}_{P}^{i}\left(t_{k}\right)-\mathbf{K}_{P}^{i-1}\left(t_{k}\right)$, where $\alpha$ and $\lambda$ are the step size and the momentum constant (for faster convergence), respectively. Go back to Step 1.

\section{NUMERICAL RESULTS}

In this section, we apply the proposed controller on two nonlinear systems to validate its effectiveness in tracking pre-defined trajectories and stabilizing the system to a fixed configuration.

\subsection{Example 1: Wheeled Mobile Robot}

In this example, we aim to solve the trajectory tracking problem of a wheeled mobile robot. The robot configuration is taken as $\mathbf{x}(t) \equiv[x(t), y(t), \theta(t)]^{T}$, where $[x(t), y(t)]^{T} \in$ $\mathbb{R}^{2}$ are the coordinates of the robot's center point on the plane, and $\theta(t) \in[-\pi, \pi)$ is its heading angle, relative to a ground-fixed inertial reference frame X-Y. The linear and angular velocities of the robot are represented by $v$ and $\omega$, respectively. At any time $t \in \mathscr{I}$, the robot kinematic model is given by

$$
\dot{\mathbf{x}}(t)=\sum_{j=1}^{2} \mathbf{g}_{j}[\mathbf{x}(t)] u_{j}(t)+\mathbf{G}[\mathbf{x}(t)] \xi(t)
$$

where $\mathbf{u}(t)=[v(t), \omega(t)]^{T}$ is the control input. The vector fields $\mathbf{g}_{1}[\mathbf{x}(t)]=[\cos \boldsymbol{\theta}, \sin \theta, 0]^{T}$ and $\mathbf{g}_{2}[\mathbf{x}(t)]=[0,0,1]^{T}$ follow assumption 1, and $\mathbf{G}[\mathbf{x}(t)]=\left[\mathbf{g}_{1}[\mathbf{x}(t)], \mathbf{g}_{2}[\mathbf{x}(t)]\right]^{T}$. The actuator noise $\xi(t) \equiv\left[\xi_{1}(t), \xi_{2}(t)\right]^{T} \in \mathscr{B}\left(\bar{\xi}, r_{d}\right)$ areis associated with the robot's linear and angular velocities. Note how (34) is similar to the system model (1) with the drift term $\mathbf{g}_{0}[\mathbf{x}(t)]=\mathbf{0}, n=3$, and $m=s=2$. The robot is set to track a feasible and smooth desired trajectory defined in the task space by $\left(x^{d}(t), y^{d}(t)\right)$, for $t \in \mathscr{I} \equiv$ $\left[\begin{array}{ll}0 & 60\end{array}\right]$ s, satisfying

$$
\dot{\mathbf{x}}^{d}(t)=\sum_{j=1}^{2} \mathbf{g}_{j}\left[\mathbf{x}^{d}(t)\right] u_{j}^{d}(t), \mathbf{x}^{d}(0)=\mathbf{x}_{0}^{d}
$$

where $\mathbf{x}^{d}(t)=\left[x^{d}(t), y^{d}(t), \theta^{d}(t)\right]^{T}, t \in \mathscr{I}$ is the desired state, and $u_{1}^{d}(t)=v^{d}(t)$ and $u_{2}^{d}(t)=\omega^{d}(t)$ are the desired linear and angular velocities. Solving for $v^{d}(t)$ and $\omega^{d}(t)$ 
from 35 yields

$$
\begin{aligned}
& v^{d}(t)= \pm \sqrt{\left[\dot{x}^{d}(t)\right]^{2}+\left[\dot{y}^{d}(t)\right]^{2}}, \\
& \theta^{d}(t)=\operatorname{ATAN} 2\left\{\frac{\dot{y}^{d}(t)}{v^{d}(t)}, \frac{\dot{x}^{d}(t)}{v^{d}(t)}\right\}, \text { and } \\
& \omega^{d}(t)=\dot{\theta}^{d}(t)=\frac{\ddot{\mathbf{y}}^{d}(t) \dot{x}^{d}(t)-\ddot{\mathbf{x}}^{d}(t) \dot{y}^{d}(t)}{\left[v^{d}(t)\right]^{2}}
\end{aligned}
$$

with codomain in all four quadrants [35]. In order to control (34) to track (35), the error state dynamic model in a rotated coordinate frame can be written as [36, 37]:

$$
\left[\begin{array}{c}
\dot{e}_{x} \\
\dot{e}_{y} \\
\dot{e}_{\theta}
\end{array}\right]=\left[\begin{array}{ccc}
0 & \omega^{d}-\omega_{e} & 0 \\
-\omega^{d}+\omega_{e} & 0 & 0 \\
0 & 0 & 0
\end{array}\right]\left[\begin{array}{c}
e_{x} \\
e_{y} \\
e_{\theta}
\end{array}\right]+\left[\begin{array}{c}
v_{e} \\
v^{d} \sin e_{\theta} \\
\omega_{e}
\end{array}\right],
$$

which is equivalent to (7). We now form the robot's feedback system as in (9) using the feedback control (8). Hence, the optimal time-varying feedback gain $\mathbf{K}_{P}^{*}(t) \in \mathscr{K}_{a d} \subset$ $\mathbb{R}^{2 \times 3}$, for $t \in \mathscr{I} \equiv[0,60] \mathrm{s}$, will drive the system (36) such that $\mathbf{e}(t) \rightarrow \mathbf{0}$, and $\tilde{\mathbf{u}}(t) \rightarrow \mathbf{0}$, for $t \in \mathscr{I}$. An exaggerated actuator noise $\xi(t) \in \mathscr{B}\left(0, r_{d}\right) \subset \mathbb{R}^{2}, t \in \mathscr{I}$, with $r_{d}=24^{\circ} \cdot \mathrm{s}^{-1}$ (see (33) $)$ is deliberately chosen to emphasize the effectiveness of the controller.

A numerical simulation was conducted to test the robot's tracking performance on an eight-shaped trajectory defined by $x^{d}(t)=\sin (\pi t / 4.8), y^{d}(t)=\sin (\pi t / 9.5)$, with an initial position and orientation of $(1,0.2) \mathrm{m}$ and $0^{\circ}$, respectively. The weight matrices of the cost functional (11) were chosen as $\mathbf{P}\left(t_{f}\right)=\mathbf{Q}(t)=\operatorname{diag}(1,1,2)$, for $t \in \mathscr{I}$. The maximum iterations for the optimization procedure was set to 50. However, more iterations would obviously yield a finer tracking performance. The results are shown in Fig. 1. It is interesting to see that the tracking errors $\left[e_{x}(t), e_{y}(t), e_{\theta}(t)\right]^{T}$ (Fig. 1(b) decayed to zero in about $3 \mathrm{~s}$ and remained practically nil for the rest of the trajectory. The initial convergence phase is essential for the controller to filter out the speed noise by selecting the optimal feedback gain $\mathbf{K}_{P}^{*}(t)$. The variation of $\mathbf{K}_{P}(t), t \in \mathscr{I}$, significantly affects the cost functional (11). Initially, the cost is relatively high due to the initial perturbation of the robot from its desired initial position and remains approximately zero once the robot reaches the desired trajectory, as expected. In each iteration, the value of $\mathbf{K}_{P}(t)$ is updated according the update rule (30) yielding monotonically decreasing Hamiltonian (14). Since the Hamiltonian (14) depends on $\mathbf{K}_{P}(t)$ and the cost function (11) is an indirect function of $\mathbf{K}_{P}$ and $\xi$, it is natural that the overall system cost $J$ (position and orientation error, in this case) gradually decreases after each iteration, yielding a better tracking performance.

\subsection{Example 2: Hopping Robot}

Now, we validate the controller's ability to regulate the posture of a hopping robot in flight mode [38, p. 366].

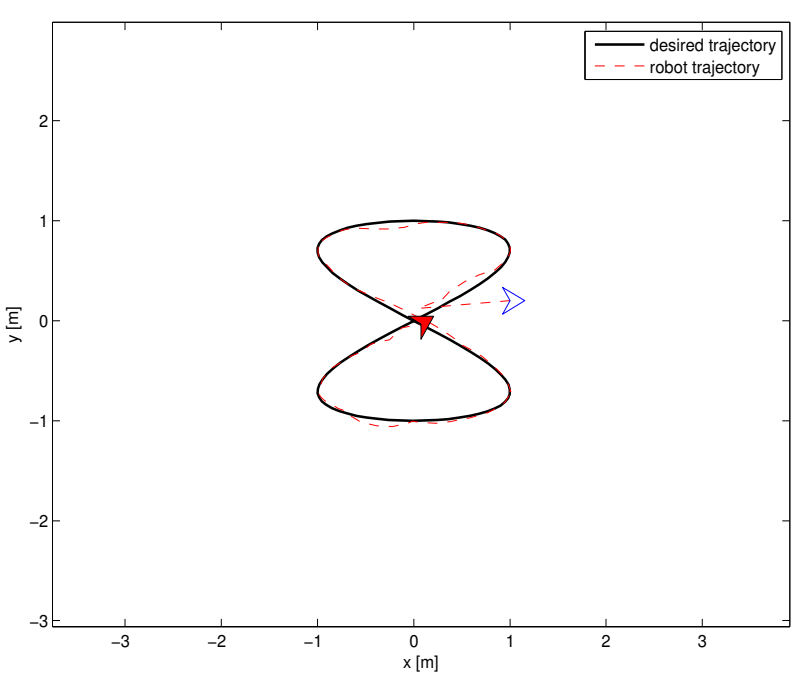

(a)

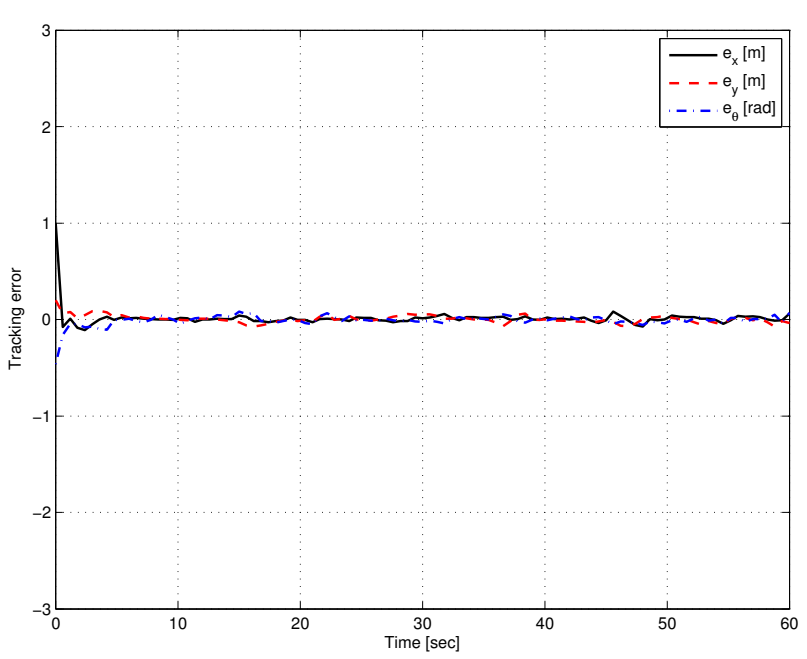

(b)

Fig 1: Tracking performance (a) eight-shaped trajectory (hollow arrow: initial pose, solid arrow: final pose) and (b) error.

The robot state is defined as $\mathbf{x} \triangleq\left[x_{1}, x_{2}, x_{3}\right]^{T}=[\psi, l+$ $1, \theta]^{T}$, where $\psi$ is the robot's hip angle with respect to its body, $l$ is the length of the leg extension, and $\theta$ is the body angle from the horizontal axis [39]. The robot's kinematic model is given by

$$
\dot{\mathbf{x}}=\mathbf{G}(\mathbf{x})(\mathbf{u}+\xi)=\left[\begin{array}{cc}
1 & 0 \\
0 & 1 \\
-\frac{x_{2}^{2}}{1+x_{2}^{2}} & 0
\end{array}\right]\left[\begin{array}{l}
u_{1}+\xi_{1} \\
u_{2}+\xi_{2}
\end{array}\right]
$$

which is in the form of $(1)$ with $\mathbf{g}_{0}(\mathbf{x})=\mathbf{0}, n=3$, and $m=s=2$. The variable $(t)$ is dropped for clarity. The control inputs are the hip angular rate $u_{1}$ and the leg extension velocity $u_{2}$. The goal is to stabilize the robot at a fixed configuration $\mathbf{x}^{d}\left(t_{f}\right)=[0,0,0]^{T}$ from an initial state $\mathbf{x}(0)=\mathbf{x}_{0}$ as $t \rightarrow t_{f}=60 \mathrm{~s}$ in the presence of process noise 
bounded by a sphere of radius $r_{d}=0.1$. Since $\mathbf{x}^{d}\left(t_{f}\right)=\mathbf{0}$, model (37) is in the form of the generalized kinematic model (9) with $\mathbf{u}(t)=\mathbf{K}_{P}(t) \mathbf{x}(t)$, where $\mathbf{u}=\left[u_{1}, u_{2}\right]^{T}$ and $\mathbf{x}=\left[x_{1}, x_{2}, x_{3}\right]^{T}$. The simulation was run with an initial

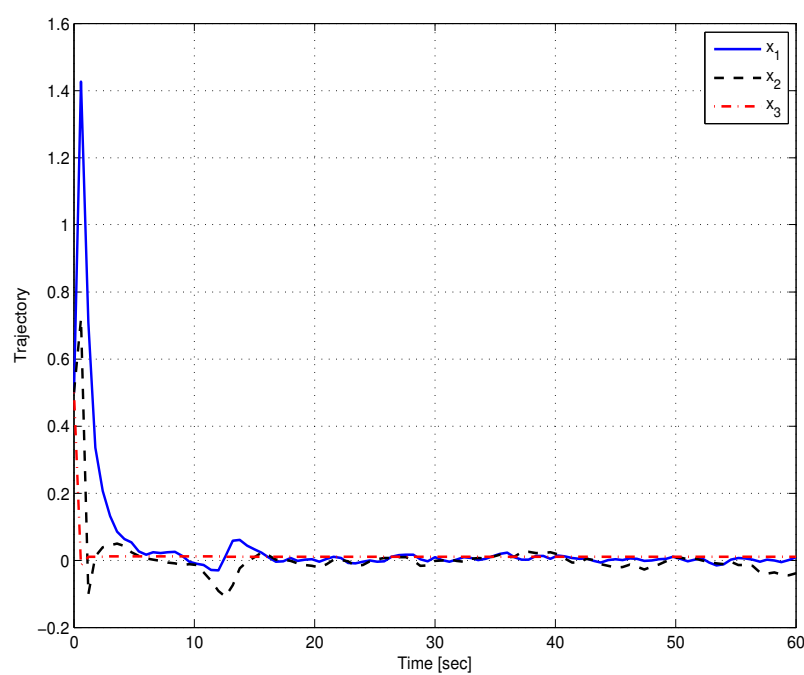

(a)

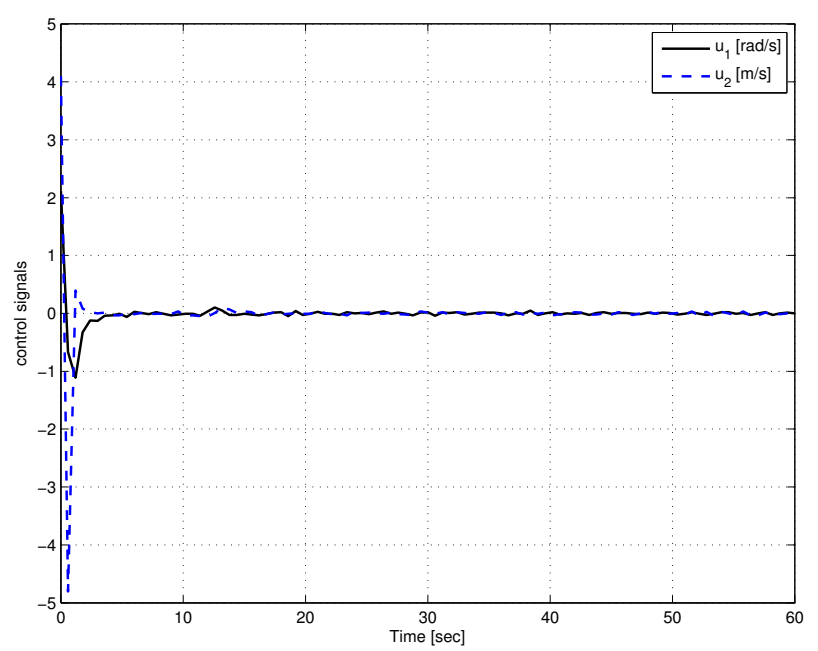

(b)

Fig 2: Stabilization performance of a hopping robot in flight phase, (a) state trajectory and (b) control signals.

state $\mathbf{x}_{0}=[0.5,0.5,0.5]^{T}$. The sampling time $T$, number of iterations for computing optimal $\mathbf{K}_{P}^{*}(t), \alpha$, and $\lambda$, are chosen as $0.6 \mathrm{~s}, 200,0.02$, and 0.1 , respectively. The weight matrices of the cost function (11) are set to $\mathbf{P}\left(t_{f}\right)=$ $20 \mathbf{Q}(t)$ and $\mathbf{Q}(t)=\operatorname{diag}(1,1,2)$. Since the goal is to stabilize the robot to a fixed configuration, this choice is driven by the fact that more weight is to be assigned to the matrix corresponding to the posture regulation, $\mathbf{P}$, than to the one controlling the tracking, $\mathbf{Q}$.

The stabilization performance of the hopping robot in flight phase is shown in Fig. 2. It took about $5 \mathrm{~s}$ for the controller to stabilize the robot to the target configura- tion (Fig. 2(a) while forcing the control signals to practically decay to zero (Fig. 2(b)) . The initial fluctuations in the state trajectory and the control signals are due to the initial posture error. The embedded process noise led to small fluctuations in both signals but didn't prevent the controller to converge to the desired posture. Since we considered the system's process noise, $\xi$, to be additive to the control inputs, it is theoretically impossible for the controller to achieve a zero steady-state error in a finite time. Furthermore, the current set of simulations considered maximum noise as they yield the worst Hamiltonian defined in 32. The control law, $\mathbf{K}_{P}^{*}(t), t \in \mathscr{I}$, was unable to tolerate noise bigger than the one chosen for simulations herein while maintaining practically zero tracking error almost everywhere. This makes sense as we claimed in section 5. that the process noise has to be chosen from the closed ball of radius $r_{d}$ (maximum).

\section{CONCLUSION}

In this paper, an optimal time-varying P-controller is proposed for simultaneously solving the state tracking and fixed-point stabilization problems of a class of nonlinear systems taking into account their input noise. Its novelty is based on optimizing the feedback gain to compute the optimal control inputs without having to linearize the system's model. Numerical simulations showed the controller's efficiency and asymptotic convergence in the face of unstructured uncertainties which didn't have to be explicitly modeled. It is worth pointing out that the same controller can also be applied to track the system's output (instead of the states) by simply replacing the state feedback gain with the output feedback gain. Note that the controller is not meant to replace the robust MPC. It rather can be regarded as an alternative MPC strategy which is capable of solving both stabilization and trajectory tracking problems for linear and nonlinear nonholonomic systems in an unified manner. It has the ability to solve these problems even for a class of semi-linear partially-observed systems.

\section{ACKNOWLEDGMENT}

The authors would like to thank Dr. N. U. Ahmed for his suggestions on the robustness study.

\section{REFERENCES}

[1] W. Luo, Y.-C. Chu, and K.-V. Ling, "Inverse optimal adaptive control for attitude tracking of spacecraft," IEEE Transactions on Automatic Control, vol. 50, no. 11, pp. 1639 - 1654, nov. 2005.

[2] I. Cervantes and J. Alvarez-Ramirez, "On the PID tracking control of robot manipulators," Systems and Control Letters, vol. 42, no. 1, pp. 37-46, Jan. 2001. 
[3] C.-S. Kim, K.-S. Hong, and M.-K. Kim, "Nonlinear robust control of a hydraulic elevator: experimentbased modeling and two-stage lyapunov redesign," Control Engineering Practice, vol. 13, no. 6, pp. 789-803, June 2005.

[4] D. Q. Wei, X. S. Luo, B. Zhang, and Y. H. Qin, "Controlling chaos in space-clamped fitzhughâĂŞnagumo neuron by adaptive passive method," Nonlinear Analysis: Real World Applications, vol. 11, no. 3, pp. 1752-1759, June 2010.

[5] M. Rehan, K.-S. Hong, and S. S. Ge, "Stabilization and tracking control for a class of nonlinear systems," Nonlinear Analysis: Real World Applications, vol. 12, no. 3, no. 3, pp. 1786-1796, 2011.

[6] S. S. Keerthi and E. G. Gilbert, "Optimal infinitehorizon feedback laws for a general class of constrained discrete-time systems: stability and movinghorizon approximations," Journal of Optimization Theory and App., vol. 57, no. 2, pp. 265-93, May 1988.

[7] C. Tarin, B. Tibken, H. Brugger, and E. Hofer, "Optimal feedback position control for an autonomous mobile robot," in Proceedings of the American Control Conference, vol. 3, pp. 1491 -1492 vol.3, 2000.

[8] P. Soueres and J. D. Boissonnat, "Optimal trajectories for nonholonomic mobile robots," in Robot Motion Planning and Control (J.-P. Laumond, ed.), vol. 229 of Lec. Notes in Control and Info. Sciences, ch. 3, pp. 93-169, Springer, 2000.

[9] L. Sheng, M. Guoliang, and H. Weili, "Stab. \& optimal control of nonhol. mob. robot," in ICARCV, vol. 2, pp. 1427-1430, dec 2004.

[10] F. Allgower, T. A. Badgwell, S. J. Qin, J. B. Rawlings, and S. J. Wright, "Nonlinear predictive control and moving horizon estimation-an introductory overview," in Advances in Control, Hightlights of ECC'99, pp. 391-449, New York: Springer, 1999.

[11] D. Q. Mayne, J. B. Rawlings, C. V. Rao, and P. O. M. Scokaert, "Constrained model predictive control: stability and optimality," Automatica, vol. 36, no. 6, pp. 789-814, June 2000.

[12] S. de Oliveira Kothare and M. Morari, "Contractive model predictive control for constrained nonlinear systems," IEEE Transactions on Automatic Control, vol. 45, no. 6, pp. 1053-1071, jun 2000.

[13] M. Diehl, H. Ferreau, and N. Haverbeke, "Efficient numerical methods for nonlinear mpc and moving horizon estimation," in Nonlinear Model Predictive Control-Towards New Challenging Applications, pp. 391-417, Springer, 2000.

[14] J. Rawlings and D. Mayne, Model Predictive Control: Theory and Design. Madison: WI: Nob Hill Publishing, 2009.

[15] K. Graichen and A. Kugi, "Stability and incremental improvement of suboptimal mpc without terminal constraints," IEEE Transactions on Automatic Control, vol. 55, no. 11, pp. 2576-2580, nov. 2010.

[16] F. A. C. C. Fontes, "Discontinuous feedbacks, discontinuous optimal controls, and continuous-time model predictive control," Intl. Jnl. of Robust and Nonlinear Control, vol. 13, no. 3, pp. 191-209, Mar. 2003.

[17] F. A. C. C. Fontes and L. Magni, "Min-max model predictive control of nonlinear systems using discontinuous feedbacks," IEEE Transactions on Automatic Control, vol. 48, no. 10, pp. 1750-1755, Oct. 2003.

[18] R. Rajamani, "Observers for lipschitz nonlinear systems," IEEE Transactions on Automatic Control, vol. 43, no. 3, pp. 397-401, mar 1998.

[19] J. Sun and G. Liu, "State feedback and output feedback control of a class of nonlinear systems with delayed measurements," Nonlinear Analysis, vol. 67, no. 5, pp. 1623-1636, Sept. 2007.

[20] V. F. Sokolov, "Adaptive suboptimal tracking for a first-order object under lipschitz uncertainty," $A u$ tomation and Remote Control, vol. 64, no. 3, pp. 457-67, Mar. 2003.

[21] V. Sokolov, "Adaptive suboptimal tracking for the first-order plant with lipschitz uncertainty," IEEE Transactions on Automatic Control, vol. 48, no. 4, pp. 607-612, april 2003.

[22] S. Liuzzo, R. Marino, and P. Tomei, "Adaptive learning control of nonlinear systems by output error feedback," IEEE Transactions on Automatic Control, vol. 52, no. 7, pp. $1232-1248$, july 2007.

[23] Z. K. Nagy and R. D. Braatz, "Open-loop and closed-loop robust optimal control of batch processes using distributional and worst-case analysis," Journal of Process Control, vol. 14, no. 1, pp. 411422, July 2004.

[24] J. B. R. Yiyang Jenny Wang, "A new robust model predictive control method i: theory and computation," Journal of Process Control, vol. 14, no. 1, pp. 231-247, Nov. 2004.

[25] A. Ailon and I. Zohar, "Control strategies for driving a group of nonholonomic kinematic mobile robots in formation along a time-parameterized path," IEEE/ASME Transactions on Mechatronics, vol. 17, no. 2, pp. 326-336, April 2012.

[26] D. Chwa, "Tracking control of differential-drive wheeled mobile robots using a backstepping-like feedback linearization," IEEE Trans. on Systems, Man and Cybernetics, Part A: Systems and Humans, vol. 40, no. 6, pp. 1285-1295, Nov. 2010.

[27] Z. Li, K. Yang, S. Bogdan, and B. Xu, "On motion optimization of robotic manipulators with strong nonlinear dynamic coupling using support area level set algorithm," International Journal of Control, Automation, and Systems, vol. 11, no. 6, pp. 12661275, December 2013. 
[28] D. Lee, H. J. Kim, and S. Sastry, "Feedback linearization vs. adaptive sliding mode control for a quadrotor helicopter," International Journal of Control, Automation, and Systems, vol. 7, no. 3, no. 3, pp. 419-428, 2009.

[29] S.-Q. Liu, J.-G. Lu, and Z.-L. Jing, "Trajectory linearization based output tracking control of an unmanned tandem helicopter with variance constraints," International Journal of Control, Automation, and Systems, vol. 8, no. 6, pp. 1257-1270, May 2010.

[30] B. Zhu and W. Huo, "Trajectory linearization control for a miniature unmanned helicopter," International Journal of Control, Automation, and Systems, vol. 11, no. 2, pp. 286-295, December 2013.

[31] H. L. Royden and P. M. Fitzpatrick, Real Analysis. Boston: Pearson Education Inc., 4th ed., 2010.

[32] H. K. Khalil, Nonlinear Systems. Upper Saddle River, New Jersey: Addison-Wesley, Pearson Education Limited,, 3rd ed., 2002.

[33] N. U. Ahmed and M. S. Miah, "Optimal feedback control law for a class of partially observed uncertain dynamic systems: A min-max problem," Dynamic Systems and Applications, vol. 20, no. 1, no. 1, pp. 149-167, 2011.

[34] N. U. Ahmed, Dynamic Systems and Control with Applications. New Jersey: World Scientific, 2006.

[35] A. D. Luca, G. Oriolo, and C. Samson, "Feedback control of a nonholonomic car-like robot," in Robot Motion Planning and Control (J.-P. Laumond, ed.), vol. 229 of Lecture Notes in Control and Information Sciences, ch. 4, pp. 170-253, Springer, 2000.

[36] Y. Kanayama, Y. Kimura, F. Miyazaki, and T. Noguchi, "A stable tracking control method for an autonomous mobile robot," in IEEE Intl. Conf. on Robotics and Automation, (Cincinnati, USA), pp. 384-9, 1990.

[37] D. Gu and H. Hu, "Receding horizon tracking control of wheeled mobile robots," IEEE Transactions on Control Systems Technology, vol. 14, no. 4, pp. 743-749, july 2006.

[38] M. W. Spong, S. Hutchinson, and M. Vidyasagar, Robot modeling and control. John Wiley \& Sons New York, 2006.

[39] F. Rehman, M. M. Ahmed, N. M. Memon, and M. Riaz, "Time-varying stabilizing control for a hopping robot model during the flight phase," in International Multitopic Conference, pp. 400-403, dec 2006.

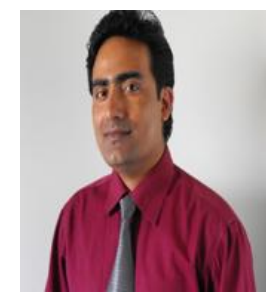

M. Suruz Miah received his bachelor of science degree in Computer Science and Engineering from Khulna University of Engineering and Technology, Bangladesh, in 2004. He earned his Master's and Ph.D. degrees in Electrical and Computer Engineering from the University of Ottawa, Canada, in 2007 and in 2012, respectively. At present, he is working for the Defence Research and Development Canada - Centre for Operational Research and Analysis (DRDC-CORA) as a Visiting Research Fellow. Miah is also a research member of the Machine Intelligence, Robotics, and Mechatronics (MIRaM) laboratory and a part-time professor at the School of Electrical Engineering and Computer Science, University of Ottawa. Miah has received numerous awards and honors including president gold medal, Alexander Graham Bell Canada Graduate Scholarship, Ontario Graduate Scholarship, and University of Ottawa Excellence Scholarship. His research interest spans the fields of mobile robotics, optimal control and estimation, distributed control, intelligent mechatronics, system identification, and Radio Frequency IDentification Technology (theory and applications).

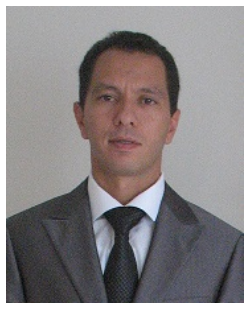

Wail Gueaieb received the Bachelor and Masters degrees in Computer Engineering and Information Science from Bilkent University, Turkey, in 1995 and 1997, respectively, and the Ph.D. degree in Systems Design Engineering from the University of Waterloo, Canada, in 2001. He is currently a Professor in the School of Electrical Engineering and Computer Science (EECS) at the University of Ottawa, Canada. He is also the founder and director of the Machine Intelligence, Robotics, and Mechatronics (MIRaM) Laboratory at EECS.

Gueaieb's research interests span the fields of intelligent mechatronics, robotics, and computational intelligence. He is the author/co-author of more than 100 patents and articles in highly reputed journals and conferences. He is currently an Associate Editor of the International Journal of Robotics and Automation. He has served as an Associate Editor, Guest Editor, and Program (co-)Chair for several international journals and conferences, such as the IEEE/ASME Transactions on Mechatronics and the IEEE Conference on Decision and Control. He has been with the industry from 2001 to 2004, where he contributed in the design and implementation of a new generation of smart automotive safety systems. 